\title{
PERSPECTIVE
}

\section{Community-acquired pneumonia as an emergency: time for an aggressive intervention to lower mortality}

\author{
S. Ewig* and A. Torres ${ }^{\#}$
}

ABSTRACT: Community-acquired pneumonia (CAP) is one of the major contemporary acute lifethreatening conditions. Short-term mortality reaches $14 \%(7 \%$ if nursing-home residents and bedridden patients are excluded) and long-term mortality reaches $50 \%$ within 5 yrs.

CAP and acute myocardial infarction (AMI) have a lot in common with regard to acuity, prognosis, need for risk stratification and early intervention, and secondary prevention measures. The obvious success in the treatment of AMI is due to an effective organisation of pre-hospital care and evidence-based interventions in the hospital within defined timescales.

Less evidence is available about effective strategies to lower short- and long-term mortality in patients with CAP. Nevertheless, it is estimated that $\sim 30 \%$ of hospitalised patients with CAP could be subject to a management approach in parallel to that of acute coronary syndrome (ACS).

Management of patients with severe CAP should be intensified using all elements that have been established in the care for patients with ACS and stroke. One of the main challenges of future research will be to define whether and which additional patients at risk of mortality truly profit from timely and structured interventions. In the meantime, patients at increased risk of death according to clinical prediction tools should also be subject to an aggressive management approach.

KEYWORDS: Community-acquired pneumonia, emergency medicine, health outcomes, health services research, respiratory infection

ommunity-acquired pneumonia (CAP) continues to be a frequent infectious condition. With an incidence of three to five cases per 1,000 persons per yr, it is a major acute disorder [1-3]. Incidence rates are up to 10fold higher in the elderly population [4]. Up to $75 \%$ of patients with CAP are hospitalised and of these, up to $10 \%$ require admission to the intensive care unit (ICU) [1, 2]. It has been estimated that $\sim 915,900$ cases of CAP occur annually among the elderly in the USA and that approximately one out of every 20 persons aged $\geqslant 85$ yrs will have a new episode of CAP each year [5]. According to a recent nationwide survey in Germany including all hospitalised patients over a period of 2 yrs, mortality reaches $14 \%$, which is nearly double the figures reported in most studies including selected patients. Mortality reached up to $30 \%$ in the elderly and disabled [2]. In accordance with a previous large US study in elderly patients [6], mortality was shown to follow an acute pattern, with most deaths occurring within the first $24 \mathrm{~h}$ of admission. This was true across all risk classes, even the lowest one. CAP is also associated with considerable excess mortality after recovery from the acute episode, reaching $50 \%$ within 5 yrs after hospital discharge. This holds true also after adjusting for age and comorbidity [7-10]. Due to general demographic trends, at least in Western societies, case numbers of CAP are expected to increase considerably [11-13]. Therefore, the relevance of CAP as a major public health challenge is clearly increasing steadily.

Despite these impressive figures, no effort has been made to organise a major public health effort in order to reduce short- and long-term mortality. Recent authoritative guidelines have, appropriately, focused on the improvement of key processes of care, including severity assessment, selection of treatment setting and differential empirical antimicrobial treatment [14-16].
AFFILIATIONS

*Thoraxzentrum Ruhrgebiet, Kliniken für Pneumologie und Infektiologie, Evangelisches Krankenhaus Herne und Augusta-Kranken-Anstalt Bochum, Bochum, Germany. " Servei de Pneumologia, Institut Clinic del Tórax, Hospital Clinic de Barcelona, Facultad de Medicina, Universitat de Barcelona, Idibaps, Ciber de Enfermedades Respiratorias, Barcelona, Spain.

CORRESPONDENCE

S. Ewig

Thoraxzentrum Ruhrgebiet

Kliniken für Pneumologie und Infektiologie

Evangelisches Krankenhaus Herne und Augusta-Kranken-Anstalt Bochum

Bergstrasse 26

44791 Bochum

Germany

E-mail: ewig@augusta-bochum.de

Received:

Dec 282010

Accepted after revision:

Feb 072011

First published online:

Feb 242011

European Respiratory Journal Print ISSN 0903-1936 Online ISSN 1399-3003 
However, recommendations remain aimed at individual care, and it appears that there is little attention paid to the real potential of preventing excess mortality. As a result, prehospitalisation times remain inadvertently long and timescales of interventions largely undefined. Moreover, apart from a recommendation for vaccination, no advice is given for the post-hospitalisation period.

The following statement is guided by the notion that CAP should be recognised as one of the major contemporary acute life-threatening conditions requiring a corresponding public health effort to improve outcomes. In fact, much can be learnt from the lessons of cardiovascular disease management. The obvious success in the treatment of acute myocardial infarction (AMI) is due to an effective organisation of pre-hospital care and evidence-based interventions in the hospital within defined timescales. CAP and AMI have much in common with regard to acuity, prognosis, need for risk stratification and early intervention, and secondary prevention measures. Evidently, these parallels are limited to the structure of management of an acute condition with high mortality and considerable potential for life-saving interventions. Moreover, less evidence is available about effective strategies to lower short- and long-term mortality. However, with these limitations in mind, nearly every element in the management of AMI could and should find its counterpart in the management of CAP.

\section{HISTORICAL PERSPECTIVE}

Up to the middle of the last century, pneumonia was recognised as a major life-threatening condition. Mortality of pneumonia was very high, reaching $100 \%$ in elderly patients with pneumococcal bacteraemia [17]. Prognosis of pneumonia complications, such as abscess formation or empyema, was poor. Standard treatment was not available, and interventions such as serum therapy yielded conflicting results. Thus, pneumonia was a regarded as a leading public health problem [18].

With the emergence of effective antimicrobial treatment, mortality of pneumonia was reduced dramatically, and the fear of pneumonia and its status as a public health emergency have been lost [18]. Pneumonia, as well as infectious diseases as a whole, were increasingly regarded as solved problems, and attention shifted to modern conditions limiting life expectancy, such as cardiovascular diseases and malignancies.

In the meantime, infectious diseases have been rediscovered as major problems, and despite considerable advances in diagnosis and treatment, infections are at the top of the agenda of conditions urgently in need of new approaches and solutions [19]. Lower respiratory tract infections (LRTIs) in particular are the leading cause of hospitalisations for infectious diseases [20].

In contrast, progress in the treatment of cardiovascular diseases is unequivocally impressive. The management of acute coronary syndromes (ACS) is perhaps one of the most obvious examples. The natural history of this acute, lifethreatening condition is associated with high mortality, yet after decades of intensive efforts, in-hospital mortality does not exceed $\sim 5 \%[21,22]$. This major progress is due to a fabulous interaction of basic research and clinical application, resulting in a myriad of excellent randomised studies with meaningful conclusions. In addition, and not least, cardiologists have succeeded in raising the awareness of ACS as an emergency with a major therapeutic life-saving potential. This was possible due to the background of convincing data showing that "time is muscle". If every minute matters, evidently, emergency medical systems linked to hospitals capable of providing a rapid diagnosis and performing the relevant interventions effectively and in time had to be created. With the identification of patients with non-ST elevation ACS (NSTEACS) at increased risk of mortality with the use of the biomarker troponin, subsequent major progress in risk stratification was made.

As a result, a generally accepted and increasingly sophisticated management algorithm continues to contribute to optimal treatment of patients with ACS. Finally, distinct interventions to identify possible complications in- and outside the hospital, as well as to address risk factors for atherosclerosis, have been applied, and these further reduce mortality from cardiovascular diseases. Similar efforts have been made in the field of stroke, following many of the key elements of the structure of ACS management ("time is brain") [23].

\section{COMPARISON OF INCIDENCE AND MORTALITY RATES OF ACS AND CAP}

The annual incidence of ACS in Europe is around one case per 80-170 persons per yr. The incidence of hospital admissions for non-ST elevated myocardial infarction (NSTEMI) has been shown to be in the range of three cases per 1,000 persons per yr in Europe, with variations among European countries. In the UK, the incidence of ST elevated myocardial infarction (STEMI) is around six cases per 1,000 persons per yr in males and two cases per 1,000 persons per yr in females.

The global mortality rate of STEMI is $\sim 40 \%$, with two-thirds occurring before hospital admission. Overall hospital mortality of STEMI is around $6-7 \%$ and that of NSTEMI is around $4-5 \%$, but at 6 months, their respective mortality rates are similar. Mortality rates of STEMI tend to be $\sim 3 \%$ higher in registries than in clinical trials. Long-term death rates in NSTEMI are even higher than in those with STEMI (11 versus 7\%) [21, 22].

Thus, incidence rates of pneumonia requiring hospitalisation are comparable to that of NSTEMI and STEMI. The excessive pre-hospital mortality is unique for STEMI. Pre-hospital mortality of pneumonia is unknown but is probably minimal; mortality of patients with mild pneumonia treated outside the hospital is low (1-3\%). However, in-hospital mortality of patients with hospitalised CAP including all risk groups is $\sim 14 \%$. If patients admitted from nursing homes and bedridden patients are excluded, it is still $\sim 7 \%$. Nursing-home residence and a bedridden condition increase mortality two- and threefold, respectively. Studies in elderly patients (usually $>65$ yrs of age) reported a mortality of $\sim 30 \%$ [2].

The risk of mortality is highly dependent on pneumonia severity. This can be assessed using clinical prediction tools. The Pneumonia Severity Index and the CURB-65 (confusion, urea $>7 \mathrm{mmol} \cdot \mathrm{L}^{-1}$, respiratory frequency $\geqslant 30$ breaths $\cdot \mathrm{min}^{-1}$, systolic blood pressure $<90 \mathrm{mmHg}$ or diastolic blood pressure $\leqslant 60 \mathrm{mmHg}$, and age $\geqslant 65 \mathrm{yrs}$ ) or CRB-65 (confusion, respiratory frequency $\geqslant 30$ breaths $\cdot \mathrm{min}^{-1}$, systolic blood pressure $<90 \mathrm{mmHg}$ or diastolic blood pressure $\leqslant 60 \mathrm{mmHg}$, and age $\geqslant 65 \mathrm{yrs}$ ) all result in predictions in a three-class pattern. Patients 
at low risk have a mortality of $1-3 \%$, at intermediate risk of 8 $12 \%$ and at high risk of up to $35 \%[2,24,25]$. Mortality rates of patients with severe CAP admitted to the ICU reach 30\% [26].

These mortality figures seem impressively high. Mortality rates of hospitalised CAP patients judged to be at intermediate risk still parallel mortality rates in patients with STEMI. Even more striking is the high excess mortality rate in survivors of CAP, reaching $\leqslant 50 \%$ within 5 yrs [7-10].

Following a 5-yr nationwide quality-assurance programme in Germany, no reduction in mortality could be documented [27]. The true potential to reduce mortality rates of CAP is unknown. In general, the incidence of pneumonia is age dependent, with the highest incidence at the extremes of age, and it is obvious that mortality rates are mainly driven by age. Even so, $\sim 75 \%$ of patients $>90$ yrs of age survive their pneumonia episode [2]. However, $\sim 85 \%$ of patients who die in hospital have not previously received ventilator support [2]. Thus, it appears that a large proportion of patients who die from CAP are judged to have CAP as a terminal event of a disabling condition.

In view of these data, the potential to reduce mortality from CAP has to be assessed in patients without restrictions in treatment escalation at increased risk according to clinical prediction tools. Probably the greatest potential to decrease mortality is in patients at increased risk but without major criteria of severity at their first presentation. Given that $\sim 50 \%$ of patients with CAP are hospitalised and that around twothirds of hospitalised patients are at intermediate or high risk, an estimated $30 \%$ of hospitalised CAP patients are subject to interventions to potentially improve outcome.

\section{COMPARISON OF MANAGEMENT STRUCTURES OF ACS AND CAP}

The structure of the management guidelines of ACS follows a strict risk stratification (table 1) [21, 22]. The symptom of acute chest pain constitutes the working diagnosis of an ACS. This working diagnosis is substantiated by a 12-lead ECG and the biomarker troponin. ACS is then further divided into three risk groups: 1) STEMI (highest risk); 2) NSTEMI, with ST or T abnormalities and/or troponin elevation (high risk); and 3) NSTEACS, without troponin elevation (i.e. unstable angina after exclusion of other reasons of chest pain; low risk). Thus, ECG and the biomarker troponin are the mainstay of risk stratification when ECG does not show ST-segment abnormalities. Low risk is only established after repeated determination of ECG and troponin.

Interventions are recommended accordingly as urgent in STEMI, early $(<72 \mathrm{~h})$ in NSTEMI with troponin elevation or elective in unstable angina.

The Global Registry of Acute Coronary Events (GRACE) risk score allows calculation of the individual risk of AMI and inhospital death and after 6 months (www.outcomes.org/grace).

Comparing the management structure of ACS and CAP according to current guidelines, striking similarities but also important differences are obvious. Symptoms of fever, cough and/or dyspnoea result in the working diagnosis of a LRTI. The presence of pneumonia is confirmed by radiography. Thus, ECG and radiography are diagnostic for the most important differentiation within the working diagnosis (i.e. AMI versus non-AMI and CAP versus non-pneumonia). In contrast, whereas the diagnosis of $\mathrm{AMI}$ is equivalent to the most severe condition within ACS, assessment of severity has yet to follow the diagnosis of CAP.

At present, risk stratification of CAP identifies three groups at risk (low risk: amulatory treatment; intermediate risk: hospitalisation; high risk: ICU or intermediate care) [14-16]. However, it is clear that a subgroup at intermediate risk (and a very small one at low risk) is at risk of early deterioration and actually at high risk of death. This subgroup should be subject to monitoring and a higher level of intervention. There is an urgent need for the identification of predictors of this subgroup. If identified, this would be the parallel group to NSTEMI.

The definition of severe pneumonia is straightforward in patients with so-called major criteria (requirement for mechanical ventilation and/or vasopressors). It is less clear how to define severe pneumonia in patients without these criteria. At present, severe CAP is best defined as requirement for ventilator support and/or fluid resuscitation and/or treatment for severe complications [28].

The role of biomarkers in CAP differs from that in ACS, in that troponin identifies patients at increased risk (i.e. NSTEMI versus unstable angina, or a condition other than ACS) whereas procalcitonin identifies patients at low risk (i.e. patients with LRTI not requiring antimicrobial treatment) [29]. Furthermore, the structure is different in that clinical scores have less impact on patient management in ACS than in CAP. This is because all patients with ACS are considered to have to be monitored until a definite diagnosis is made, and because the biomarker troponin determines both diagnosis (of NSTEMI versus unstable angina) and risk stratification. The role of biomarkers in risk stratification of CAP is less well established, whereas clinical scores yield excellent predictions of the risk of death. Current data indicate that a combination of a clinical score with a biomarker will yield the best predictions [30, 31].

Notably, the recent development of a highly sensitive troponin-T assay represents a further refinement in the identification of patients with NSTEACS at increased risk, with high precision even in the 99th percentile $[32,33]$.

\section{COMPARISON OF MANAGEMENT STRUCTURES OF STEMI AND CAP}

The hallmarks of the management of STEMI include the following six issues (table 2).

\section{First medical contact and emergency care flow}

The first medical contact and emergency care flow is thought to the shorten time from suspected diagnosis to hospitalisation, which is particularly relevant in AMI due to the high prehospital mortality of $\leqslant 50 \%$. The crucial issue is to establish an emergency medical system linked to capable hospitals.

A working diagnosis of AMI has to be established as quickly as possible based on history, clinical symptoms and a 12-lead ECG: all of which are rapidly available measurements without relevant risk of a diagnostic delay. Clinical chemistry is not awaited in order to save time. 


\begin{tabular}{|c|c|c|c|}
\hline \multirow[t]{3}{*}{ TABLE 1} & e structure of management of & ute coronary syndrome $(f$ & and community-acquired pneumonia \\
\hline & \multirow[t]{2}{*}{ ACS } & \multicolumn{2}{|r|}{ CAP } \\
\hline & & Current practice & Future model \\
\hline Symptoms & Chest pain & Fever & Fever \\
\hline Syndrome & ACS & LRTI & LRTI \\
\hline Working diagnosis & Plus 12-lead ECG & Chest radiography & Chest radiography \\
\hline \multicolumn{4}{|l|}{ Risk stratification } \\
\hline \multicolumn{4}{|l|}{ Tools } \\
\hline Short-term & Biomarker (troponin) & Clinical score & Clinical score \\
\hline \multirow[t]{4}{*}{ Risk groups } & STEMI & Severe pneumonia requiring $\mathrm{ICU}$ & $\begin{array}{l}\text { Severe pneumonia requiring ventilator } \\
\text { support and/or fluid resuscitation and/or } \\
\text { treatment for severe complication }\end{array}$ \\
\hline & NSTEMI (troponin-positive) & Not defined & $\begin{array}{l}\text { Severe pneumonia requiring monitoring } \\
\text { (ICU or IMC) }\end{array}$ \\
\hline & $\begin{array}{l}\text { NSTEACS (troponin-negative, } \\
\text { unstable angina) }\end{array}$ & $\begin{array}{l}\text { Nonsevere pneumonia requiring } \\
\text { hospitalisation }\end{array}$ & Nonsevere pneumonia requiring hospitalisation \\
\hline & & $\begin{array}{l}\text { Nonstable pneumonia requiring } \\
\text { ambulatory care }\end{array}$ & $\begin{array}{l}\text { Nonsevere pneumonia requiring } \\
\text { ambulatory care }\end{array}$ \\
\hline Intervention & $\begin{array}{l}\text { STEMI: immediate } \\
\text { revascularisation }\end{array}$ & $\begin{array}{l}\text { Severe CAP: antibiotics plus } \\
\text { ventilator support and/or fluid } \\
\text { resuscitation and/or treatment of } \\
\text { severe complication }\end{array}$ & $\begin{array}{l}\text { Severe CAP: antibiotics plus ventilator } \\
\text { support and/or fluid resuscitation and/or } \\
\text { treatment of severe complication }\end{array}$ \\
\hline
\end{tabular}

LRTI: lower respiratory tract infection; GRACE: Global Registry of Acute Coronary Events; TIMI: thrombolysis in myocardial infarction; IL: interleukin; STEMI: ST elevated myocardial infarction; NSTEMI: non-ST elevated myocardial infarction; ICU: intensive care unit; IMC: intermediate care; NSTEACS: non-ST elevated ACS.

No emergency medical system is established for severe CAP. The emergency care flow is similar in severe CAP; however, the relevance of making a rapid diagnosis is not generally settled, particularly in those patients without septic shock.

\section{Pre-hospital and early in-hospital care}

A working diagnosis and specific treatment is made outside the hospital if rapid hospitalisation is not within reach. Most patients will receive reperfusion therapy by percutaneous coronary intervention and/or fibrinolysis within a time frame of $\leqslant 2 \mathrm{~h}$. A door-to-needle time of $<30 \mathrm{~min}$ and door-toballoon time of $<2 \mathrm{~h}$ are defined targets that have to be met.

Possible acute complications (pump failure and shock, mechanical complications, arrhythmias and conduction disturbances) are addressed and prophylactic treatments are listed.

To date, even parts of the guidelines for severe sepsis and septic shock are not generally imported in guidelines for severe CAP, and even less in general practice. A major issue is the incorporation of early goal-directed therapy in patients presenting with CAP and severe sepsis. There is major evidence that clinical failures are related to severe sepsis [34] and that prognosis of patients with septic shock worsens with every hour of delay in appropriate antimicrobial treatment [35], and the importance of rapid initiation of appropriate antimicrobial treatment parallels that in acute bacterial meningitis [36].

No exact door-to-antibiotic timescale has been defined in guidelines or settled in clinical practice. The same is true for the initiation of aggressive fluid resuscitation in severe CAP with severe sepsis [37]. The evidence in favour of a door-toantibiotic time in patients without septic shock is less clear [38-42]. It probably heavily depends on the populations studied. Also, most studies are heavily underpowered to find a small but relevant difference in mortality. Some authors have even raised concern about such a timescale because of the risk of overtreatment [43-45]. However, overtreatment is only a concern if antimicrobial treatment is inappropriately administered prior to a clear diagnosis. In fact, the risk of inadequate 
TABLE 2 Comparison of the structure of management of ST elevated myocardial infarction (STEMI) and severe communityacquired pneumonia (CAP)

Severe CAP

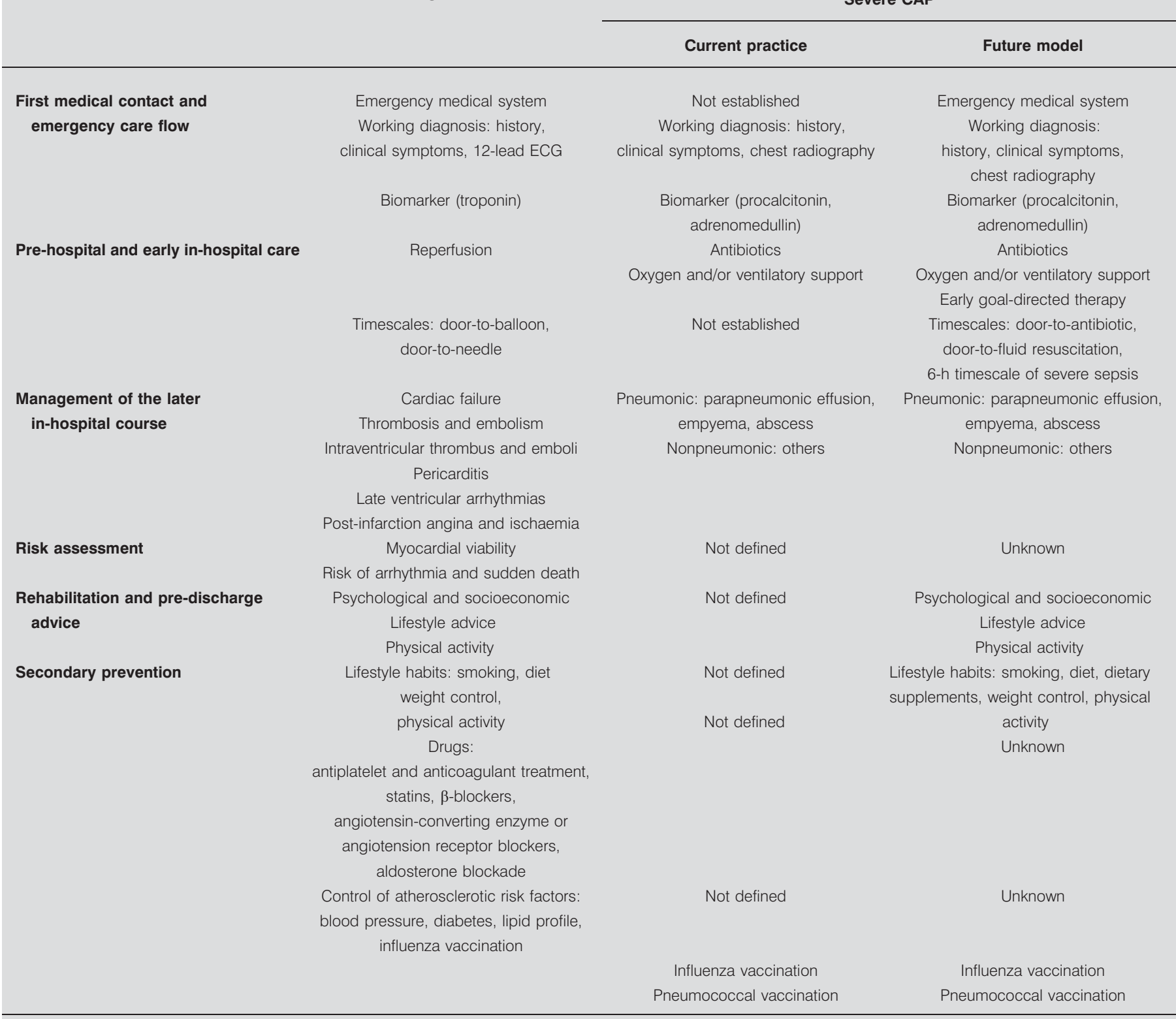

diagnosis can be eliminated if there is a pathway that ensures rapid diagnostic work-up. There is a good reason to establish immediate antimicrobial treatment within a defined timescale after the diagnosis of CAP has radiographically been confirmed [38, 39, 46, 47]. However, the exact duration of the timescale is not yet determined. It predictably heavily depends on the severity of CAP at admission.

\section{Management of in-hospital course}

This includes all measures against late complications during hospitalisation (thrombosis and embolism, intraventricular thrombus and emboli, pericarditis, late ventricular arrhythmias, post-infarction angina and ischaemia). There are parallels of these in severe CAP, such as pneumonic and nonpneumonic complications. However, they have not been addressed in a comparable systematic way in the guidelines of CAP, and are probable not routinely assessed in clinical practice. As a result, parapneumonic complicated effusions and empyemas are frequently diagnosed with delay.

\section{Risk assessment after AMI}

The sequelae of AMI are assessed (myocardial viability and risk of arrhythmia or sudden death). The assessment of risk after severe CAP has only just begun to be studied [48]. Excess 
mortality, specifically of survivors of severe CAP after discharge from hospital, has not yet been defined, but is suspected to be high in view of the excess mortality of the whole population of CAP survivors. Thus, there is a good reason to start investigations in order to identify tools for preventative interventions in patients at risk of excess mortality after severe CAP.

\section{Rehabilitation and pre-discharge advice}

Rehabilitation and pre-discharge advice include psychological and socioeconomic aspects, lifestyle advice and physical activity. Compared with STEMI, it is less clear how and to what extent severe CAP is a consequence of defined psychological and socioeconomic determinants, and even less so how these could be subject to intervention. However, it is possible that this is due to a scientific underestimation of severe CAP as a condition with psychological and socioeconomic implications. Likewise, whereas consequences of limited fitness after myocardial infarction have attracted considerable research and care efforts, no such attention has been paid to severe CAP as a condition possibly limiting physical and psychosocial activities.

\section{Secondary prevention}

Secondary prevention targets the following sections: 1) lifestyle habits (smoking, diet, weight control and physical activity); 2) drugs (antiplatelet and anticoagulant treatment, statins, $\beta$-blockers, angiotensin-converting enzyme or angiotension receptor blockers, and aldosterone blockade); 3) control of atherosclerotic risk factors (blood pressure, diabetes and lipid profile); and 4) influenza vaccination.

Interventions in lifestyle habits have not been investigated in survivors of severe CAP, although it is quite evident that these would be of equal importance in these patients as in survivors of STEMI. There are no comparable interventions aimed at risk reduction after STEMI using drugs protective to the heart or the control of risk factors for progression of atherosclerosis for survivors of severe $\mathrm{CAP}$, and it is questionable whether such interventions can be defined in the future. The only clear parallel is advice for vaccination, which includes influenza and pneumococcal vaccination in severe CAP.

\section{REQUIREMENTS FOR FUTURE PRACTICE AND RESEARCH}

On comparing the management structure of ACS and STEMI with severe CAP, strong parallels are evident. At the same time, several differences hint at important gaps in the current management of patients with severe CAP.

The management of ACS is based on an emergency medical system defining the structure of the first medical contact and emergency care flow. This is based on an awareness of a lifethreatening condition, and the importance of predefined treatment algorithms and adherence to defined timescales. No comparable awareness is evident in the care of pneumonia patients, and no comparable emergency medical system has been launched for pneumonia.

The most important gap in the risk stratification of CAP seems to be the lack of predictors for patients at increased risk (i.e. a correlate to NSTEMI with elevated troponin). At present, it appears improbable that a biomarker alone will resolve this issue; instead, a clinical prediction tool or a combination of such a tool with a biomarker are likely to provide a definition in the near future. In the meantime, it is important to keep in mind that an important group of patients apparently at moderate or even low risk are, in fact, at high risk. In the absence of a correlate to NSTEMI with elevated troponin, it seems most practical to provide increased monitoring to all hospitalised patients with increased risk scores. This inherently oversensitive approach is in parallel to the most recent trends in the management of ACS after the introduction of the highly sensitive biomarker troponin, increasing sensitivity at the cost of specificity [49].

Accordingly, a striking gap is evident with regard to the lack of timescales in the management of severe CAP. Door-toantibiotic and door-to-fluid resuscitation times for patients with CAP and septic shock, and the 6-h timescale for early goal-directed treatment in patients with CAP and severe sepsis are evidence-based goals and should urgently be established. The impact of door-to-antibiotic time in patients without shock has not been sufficiently addressed in different severity classes at presentation, but there is little risk of harm if it is applied in patients with an established diagnosis of CAP.

In-hospital management may be improved when the potential for pneumonic and nonpneumonic complications is assessed more systematically in each individual. Important areas of research should focus on the impact of psychological, socioeconomic and lifestyle implications of severe CAP, possibly leading to a more evidence-based rehabilitation programme and pre-discharge advice.

Risk factors for excess mortality in survivors of CAP and severe CAP that could be subject for intervention are not known. Correlates for a careful risk assessment in terms of myocardial viability, risk of arrhythmia and sudden death are, therefore, not defined for CAP. In view of high excess mortality rates in survivors of CAP, this is a major tool for future research. Likewise, there is a lack of correlates for interventions to prevent recurrent ACS. Since an episode of CAP poses the patient at risk for a subsequent episode, comparable research efforts should be made to identify risk factors for recurrent pneumonia and possible interventions.

Improving the effectiveness of care delivery will need to address organisational change and quality improvement programmes. Strategic improvement initiatives to produce system-level results in quality improvement have been published, which may orientate such approaches [50]. Quality performance indicators for CAP in US hospitals have already been used successfully [51]. The definition and implementation of acute care bundles for CAP are particularly promising [52].

\section{CONCLUSIONS}

In 1892, Sir William Osler wrote in his classic book Principles and Practices of Medicine that pneumonia was "the captain of men of death" [53]. Despite the availability of causal treatment with antibiotics since 1955, this premonitory sentence still holds true. However, this does not mean that no progress has been made. Instead, mortality seems to remain constant despite increasing comorbidity [54]. Nevertheless, it appears that no sufficient systematic effort has been made to substantially reduce the continuing high mortality from CAP. 
In order to accomplish this target, it is mandatory to address the research priorities listed in this article. At the same time, management of patients with severe CAP should be intensified by all classical elements that are well established in the care for patients with ACS and stroke. These include: 1) immediate emergency medical service (EMS) contact and priority EMS dispatch; 2) priority transport with advanced notification to the next hospital, ideally to a hospital with defined pre-hospital and in-hospital pathways of treatment; 3) immediate emergency room triage within defined timescales; and 4) consideration of helicopter transfer and telemedicine in rural areas in order to improve access of treatment. One of the main challenges will be to define whether and which additional patients at risk of mortality truly profit from timely and structured interventions.

The time has come to define CAP as an emergency. The slogan for CAP corresponding to ACS and stroke is "time is life". Since CAP has not been realised as such to date, educational programmes to increase awareness of CAP as an emergency among professionals (paramedics, physicians and nurses) and at the population level are mandatory.

\section{STATEMENT OF INTEREST}

None declared.

\section{ACKNOWLEDGEMENTS}

The authors are indebted to X. Bosch (Servei de Cardiologia, Institut del Tòrax, Hospital Clinic Barcelona, Barcelona, Spain) for insightful discussions and critical review of the manuscript.

\section{REFERENCES}

1 Almirall J, Bolíbar I, Vidal J, et al. Epidemiology of communityacquired pneumonia in adults: a population-based study. Eur Respir J 2000; 15: 757-763.

2 Ewig S, Birkner N, Strauss R, et al. New perspectives on community-acquired pneumonia in 388406 patients. Results from a nationwide mandatory performance measurement programme in healthcare quality. Thorax 2009; 64: 1062-1069.

3 Ochoa-Gondar O, Vila-Córcoles A, de Diego C, et al. The burden of community-acquired pneumonia in the elderly: the Spanish EVAN-65 study. BMC Public Health 2008; 8: 222.

4 Marston BJ, Plouffe JF, File TM Jr, et al. Incidence of communityacquired pneumonia requiring hospitalization. Results of a population-based active surveillance Study in Ohio. The Community-Based Pneumonia Incidence Study Group. Arch Intern Med 1997; 157: 1709-1718.

5 Jackson ML, Neuzil KM, Thompson WW, et al. The burden of community-acquired pneumonia in seniors: results of a populationbased study. Clin Infect Dis 2004; 39: 1642-1650.

6 Kaplan V, Angus DC, Griffin MF, et al. Hospitalized communityacquired pneumonia in the elderly: age- and sex-related patterns of care and outcome in the United States. Am J Respir Crit Care Med 2002; 165: 766-772.

7 Johnstone J, Eurich DT, Majumdar SR, et al. Long-term morbidity and mortality after hospitalization with community-acquired pneumonia: a population-based cohort study. Medicine (Baltimore) 2008; 87: 329-334.

8 Johnstone J, Eurich DT, Minhas JK, et al. Impact of the pneumococcal vaccine on long-term morbidity and mortality of adults at high risk for pneumonia. Clin Infect Dis 2010; 51: 15-22.

9 Bordon J, Wiemken T, Peyrani P, et al. Decrease in long-term survival for hospitalized patients with community-acquired pneumonia. Chest 2010; 138: 279-283.
10 Cecere LM, Rubenfeld GD, Park DR, et al. Long-term survival after hospitalization for community-acquired and healthcare-associated pneumonia. Respiration 2010; 79: 128-136.

11 Welte T, Torres A, Nathwani D. Clinical and economic burden of community-acquired pneumonia among adults in Europe. Thorax 2010; [Epub ahead of print DOI: 10.1136/thx.2009.129502].

12 File TM Jr, Marrie TJ. Burden of community-acquired pneumonia in North American adults. Postgrad Med 2010; 122: 130-141.

13 Isturiz RE, Luna CM, Ramirez J. Clinical and economic burden of pneumonia among adults in Latin America. Int J Infect Dis 2010; 14: e852-e866.

14 Woodhead M, Blasi F, Ewig S, et al. Guidelines for the management of adult lower respiratory tract infections. Eur Respir J 2005; 26: 1138-1180.

15 Lim WS, Baudouin SV, George RC, et al. BTS guidelines for the management of community acquired pneumonia in adults: update 2009. Thorax 2009; 64: Suppl. 3, iii1-iii55.

16 Mandell LA, Wunderink RG, Anzueto A, et al. Infectious Diseases Society of America/American Thoracic Society consensus guidelines on the management of community-acquired pneumonia in adults. Clin Infect Dis 2007; 44: Suppl. 2, S27-S72.

17 Singer M, Nambiar S, Valappil T, et al. Historical and regulatory perspectives on the treatment effect of antibacterial drugs for community-acquired pneumonia. Clin Infect Dis 2008; 47: Suppl. 3, S216-S224.

18 Podoslky S. Pneumonia Before Antibiotics: Therapeutic Evolution and Evaluation in Twentieth-Century America. Baltimore, The Johns Hopkins University Press, 2006.

19 Binder S, Levitt AM, Sacks JJ, et al. Emerging infectious diseases: public health issues for the 21st century. Science 1999; 284: 1311-1313.

20 Christensen KL, Holman RC, Steiner CA, et al. Infectious disease hospitalizations in the United States. Clin Infect Dis 2009; 49: 1025-1035.

21 Bassand JP, Hamm CW, Ardissino D, et al. Guidelines for the diagnosis and treatment of non-ST-segment elevation acute coronary syndromes. Task Force for Diagnosis and Treatment of Non-ST-Segment Elevation Acute Coronary Syndromes of European Society of Cardiology. Eur Heart J 2007; 28: 1598-1660.

22 Van de Werf F, Bax J, Betriu A, et al. Management of acute myocardial infarction in patients presenting with persistent ST-segment elevation: the Task Force on the Management of ST-Segment Elevation Acute Myocardial Infarction of the European Society of Cardiology. Eur Heart J 2008; 29: 2909-2945.

23 European Stroke Organisation. Guidelines for Stroke Management. www.eso-stroke.org/recommendations.php?cid $=9 \&$ sid $=1$ Date last accessed: February 1, 2011. Date last updated: January 2009.

24 Fine MJ, Auble TE, Yealy DM, et al. A prediction rule to identify low-risk patients with community-acquired pneumonia. $N$ Engl J Med 1997; 336: 243-250.

25 Lim WS, Lewis S, Macfarlane JT. Severity prediction rules in community acquired pneumonia: a validation study. Thorax 2000; 55: 219-223.

26 Liapikou A, Ferrer M, Polverino E, et al. Severe communityacquired pneumonia: validation of the Infectious Diseases Society of America/American Thoracic Society guidelines to predict an intensive care unit admission. Clin Infect Dis 2009; 48: 377-385.

27 BQS Institut für Qualität und Patientensicherheit. Ambulant erworbene Pneumonie. [Hospitalised community-acquired pneumonia]. www.bqs-qualitaetsreport.de/2008/ergebnisse/leistungsbereiche/pneumonie/index_html Date last accessed: February 1, 2011. Date last updated: June 30, 2009.

28 Ewig S, Woodhead M, Torres A. Towards a sensible comprehension of severe community-acquired pneumonia. Intensive Care Med 2011; 37: 214-223. 
29 Christ-Crain M, Opal SM. Clinical review: the role of biomarkers in the diagnosis and management of community-acquired pneumonia. Crit Care 2010; 14: 203.

30 Krüger S, Ewig S, Marre R, et al. Procalcitonin predicts patients at low risk of death from community-acquired pneumonia across all CRB-65 classes. Eur Respir J 2008; 31: 349-355.

31 Menéndez R, Martínez R, Reyes $S$, et al. Biomarkers improve mortality prediction by prognostic scales in community-acquired pneumonia. Thorax 2009; 64: 587-591.

32 Reichlin T, Hochholzer W, Bassetti S, et al. Early diagnosis of myocardial infarction with sensitive cardiac troponin assays. N Engl J Med 2009; 361: 858-867.

33 Keller T, Zeller T, Peetz D, et al. Sensitive troponin I assay in early diagnosis of acutemyocardial infarction. N Engl J Med 2009; 361: 868-877.

34 Aliberti S, Amir A, Peyrani P, et al. Incidence, etiology, timing, and risk factors for clinical failure in hospitalized patients with community-acquired pneumonia. Chest 2008; 134: 955-962.

35 Kumar A, Roberts D, Wood KE, et al. Duration of hypotension before initiation of effective antimicrobial therapy is the critical determinant of survival in human septic shock. Crit Care Med 2006; 34: 1589-1596

36 Aronin SI, Peduzzi P, Quagliarello VJ. Community-acquired bacterial meningitis: risk stratification for adverse clinical outcome and effect of antibiotic timing. Ann Intern Med 1998; 129: 862-869.

37 Rivers E, Nguyen B, Havstad S, et al. Early goal-directed therapy in the treatment of severe sepsis and septic shock. N Engl J Med 2001; 345: 1368-1377.

38 Meehan TP, Fine MJ, Krumholz HM, et al. Quality of care, process, and outcomes in elderly patients with pneumonia. JAMA 1997; 278: 2080-2084.

39 Houck PM, Bratzler DW, Nsa W, et al. Timing of antibiotic administration and outcomes for Medicare patients hospitalized with community-acquired pneumonia. Arch Intern Med 2004; 164: 637-644.

40 Silber SH, Garrett C, Singh R, et al. Early administration of antibiotics does not shorten time to clinical stability in patients with moderate-to-severe community-acquired pneumonia. Chest 2003; 124: 1798-1804.

41 Marrie TJ, Wu L. Factors influencing in-hospital mortality in community-acquired pneumonia: a prospective study of patients not initially admitted to the ICU. Chest 2005; 127: 1260-1270.

42 Bruns $\mathrm{AH}$, Oosterheert JJ, Hustinx WN, et al. Time for first antibiotic dose is not predictive for the early clinical failure of moderate-severe community-acquired pneumonia. Eur J Clin Microbiol Infect Dis 2009; 28: 913-919.

43 Kanwar M, Brar N, Khatib R, et al. Misdiagnosis of communityacquired pneumonia and inappropriate utilization of antibiotics: side effects of the 4-h antibiotic administration rule. Chest 2007; 131: 1865-1869.

44 Welker JA, Huston M, McCue JD. Antibiotic timing and errors in diagnosing pneumonia. Arch Intern Med 2008; 168: 351-356.

45 Pines JM, Isserman JA, Hinfey PB. The measurement of time to first antibiotic dose for pneumonia in the emergency department: a white paper and position statement prepared for the American Academy of Emergency Medicine. J Emerg Med 2009; 37: 335-340.

46 Battleman DS, Callahan M, Thaler HT. Rapid antibiotic delivery and appropriate antibiotic selection reduce length of hospital stay of patients with community-acquired pneumonia: link between quality of care and resource utilization. Arch Intern Med 2002; 162: 682-688.

47 Garnacho-Montero J, García-Cabrera E, Diaz-Martín A, et al Determinants of outcome in patients with bacteraemic pneumococcal pneumonia: importance of early adequate treatment. Scand J Infect Dis 2010; 42: 185-192.

48 Yende S, D'Angelo G, Kellum JA, et al. Inflammatory markers at hospital discharge predict subsequent mortality after pneumonia and sepsis. Am J Respir Crit Care Med 2008; 177: 1242-1247.

49 Thygesen K, Mair J, Katus H, et al. Recommendations for the use of cardiac troponin measurement in acute cardiac care. Eur Heart $J$ 2010; 31: 2197-2204.

50 Nolan TW. Execution of Strategic Improvement Initiatives to Produce System- Level Results. IHI Innovation Series White Paper. Cambridge, Institute for Healthcare Improvement, 2007.

51 Williams SC, Schmaltz SP, Morton DJ, et al. Quality of care in US hospitals as reflected by standardized measures, 2002-2004. N Engl J Med 2005; 353: 255-264.

52 Scottish Medicines Consortium. Community Acquired Pneumonia (SNAP-CAP) Quality Improvement Project. www.scottishmedicines. org.uk/SAPG/SNAP-CAP/ Date last accessed: May 16, 2011. Date last updated: June 2008.

53 Osler W. The Principles and Practice of Medicine: Designed for the Use of Practitioners and Students of Medicine. New York, D. Appleton and Company, 1892.

54 Fry AM, Shay DK, Holman RC, et al. Trends in hospitalizations for pneumonia among persons aged 65 years or older in the United States, 1988-2002. JAMA 2005; 294: 2712-2719. 